\title{
AUTOMATIC ROAD GAP DETECTION USING FUZZY INFERENCE SYSTEM
}

\author{
M. Hashemi ${ }^{\text {a }}$, M. J. Valadan Zoej ${ }^{\text {b }}$, M. Mokhtarzadeh ${ }^{\mathrm{c}}$ \\ Faculty of Geodesy and Geomatics Engineering, K.N.Toosi.University of Technology, Vali-e-asr St., Mirdamad \\ Cross, Tehran, Iran, P.C. 1996715433 - (moh.hashemi, mokhtarzadeh)@yahoo.com, valadanzouj@kntu.ac.ir
}

Commission IV, WG IV/2

KEY WORDS: Gap Detection, Road network, Fuzzy Inference System

\begin{abstract}
:
Automatic feature extraction from aerial and satellite images is a high-level data processing which is still one of the most important research topics of the field.

In this area, most of the researches are focused on the early step of road detection, where road tracking methods, morphological analysis, dynamic programming and snakes, multi-scale and multi-resolution methods, stereoscopic and multi-temporal analysis, hyper spectral experiments, are some of the mature methods in this field. Although most researches are focused on detection algorithms, none of them can extract road network perfectly. On the other hand, post processing algorithms accentuated on the refining of road detection results, are not developed as well.

In this article, the main is to design an intelligent method to detect and compensate road gaps remained on the early result of road detection algorithms. The proposed algorithm consists of five main steps as follow:

1) Short gap coverage:

In this step, a multi-scale morphological is designed that covers short gaps in a hierarchical scheme.

2) Long gap detection:

In this step, the long gaps, could not be covered in the previous stage, are detected using a fuzzy inference system. for this reason, a knowledge base consisting of some expert rules are designed which are fired on some gap candidates of the road detection results.

3) Long gap coverage:

In this stage, detected long gaps are compensated by two strategies of linear and polynomials for this reason, shorter gaps are filled by line fitting while longer ones are compensated by polynomials.

4) Accuracy assessment:

In order to evaluate the obtained results, some accuracy assessment criteria are proposed. These criteria are obtained by comparing the obtained results with truly compensated ones produced by a human expert.

The complete evaluation of the obtained results whit their technical discussions are the materials of the full paper.
\end{abstract}

\section{INTRODUCTION}

Automatic urban feature extraction from aerial and satellite images, is one of important subjects in photogrammetry and remote sensing. Roads and buildings are the most important features in urban area. Lately two decades, most of the researches are focused on road detection from remotely sensed images. (Mena 2003) has been presented a good literature review of current algorithms for road detection. However these approaches are imperfect, none of them can extract road network perfectly. Many reasons such as algorithm weakness, sensor geometry and occlusions have a bad effect on the performance of these algorithms. There must be a post-processing stage in road detection process to solve these problems.

In this research, we used the effective parameters for gap detection in a fuzzy inference system to overcome these problems.

\section{SOME BASIC DEFINITIONS}

In this section we defined some necessary concepts for better understanding.

Salient points: end point, end neighbour point and vertex in a road path are called salient point.

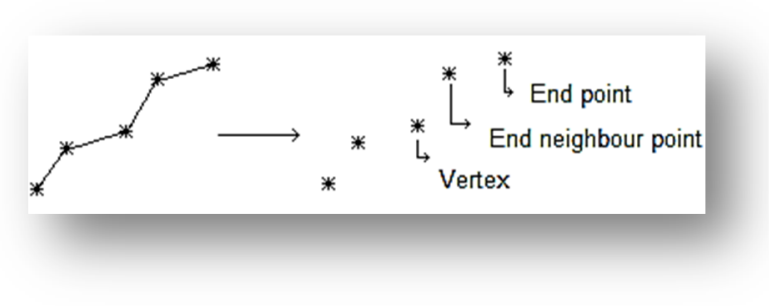

Figure 1. Salient points

Link hypothesis: the possibility of gap occurrence between tow end points is link hypothesis and it could be true or false.

Short gaps: the gaps that their lengths are shorter than the width of the road are called short gaps. 
Long gaps: the gaps that are greater than short gaps are introduced as long gaps.

Momentum: in this study, the length of each road segment is identified as momentum.

Road predictor: a model that continues the road after its end point is called road predictor. It could be a polynomial, extended linear models or kalman filter.

Gap path: in each hypothesis link a polynomial degree of four that pass throw two end points and their end neighbour points, is called gap path.

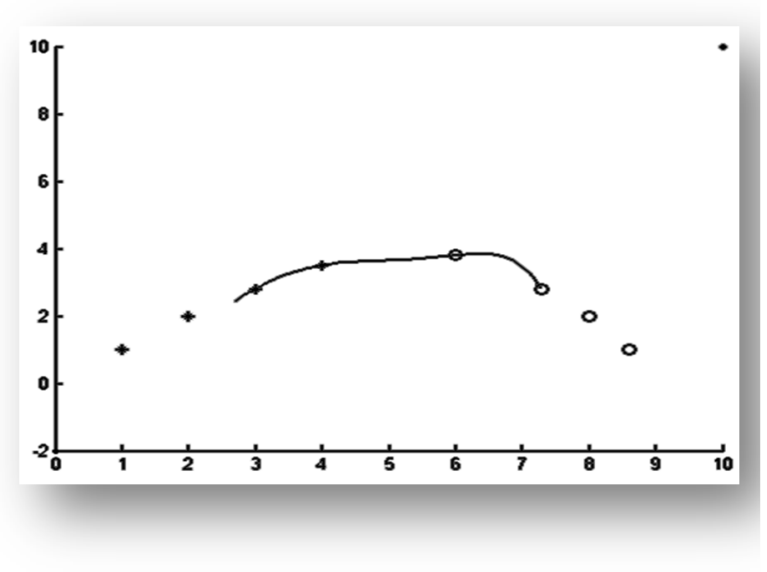

Figure 2. Gap path

Correlation: the mount of accordance between two continued path from the end points of two road segments.

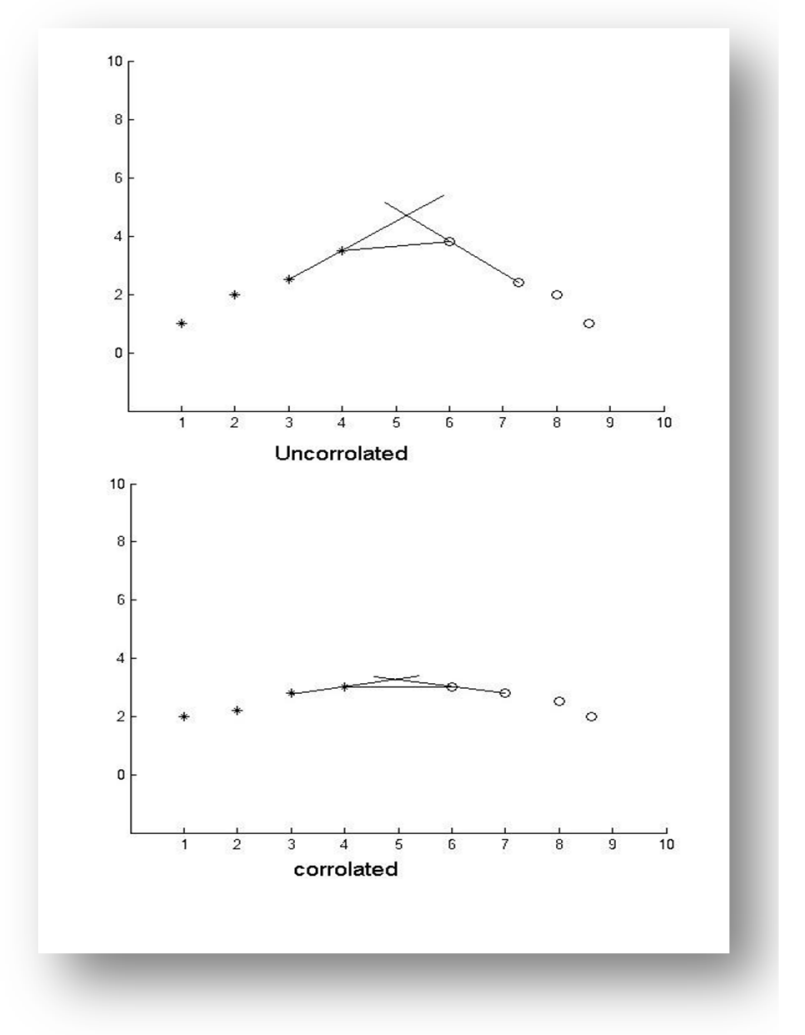

Figure 3. Correlation

\section{METHODOLOGY}

One of the advantages of this study was classifying the gaps into separate classes. Hence we classified the gaps into two major classes:

1. Short gaps

\section{Long gaps}

First of all, we used the neighbourhood concept to clean the image from short gaps.

Different view to the neighbourhood is an innovation in our work. In this regard, with analyse the neighbourhood condition in multi-scale levels. The performance of mathematical morphology in short gaps cleaning is raised. Then, using skeleton operator, the salient points and the type of them can be extract from the road network. In the next step, we choose the effective parameters in gap detection. Then with using them in a fuzzy inference system, we process the entire link hypothesis between all the end points. Each pair of points that have the better condition for connecting, are connected by gap path. In last section we discussed the performance of this system.

\subsection{Short gap coverage:}

Using morphological operators is the most common approach for cleaning binary images results from a road detection algorithm. Therefore by using a cleaning operator, we can eliminate single-pixel or multi-pixel noises laid on the surface of an image. Because dilation and erosion import some unwelcome changes into an image, they don't use directly.
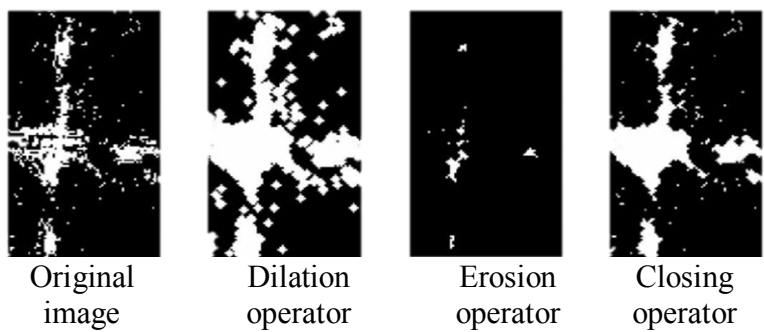

Figure 4. effects of morphological operators on binary image

Since closing operator has a good ability to keep the original size of the road while connecting adjacent segments it is a suitable operator. But we have a problem because of using permanent structural element during the work. Naturally, in roads with wider width we have an opportunity to cover the short gaps with longer lengths. (Hashemi 2011a) overcome to this issue by an multiresolution morphological algorithm. By using cleaning and closing in multi-resolution levels of the image and combining the results, the performance of mathematical morphology in covering short gaps are raised significantly. In this study, first of all, roads are classified based on their width. In the next step, cleaning and closing operate on which level of scale that road has 1 to 3 pixels width. In the last step results in all levels are combined with each other and make the final result. The results of this approach shows significant performance of it in compare with former approaches. 

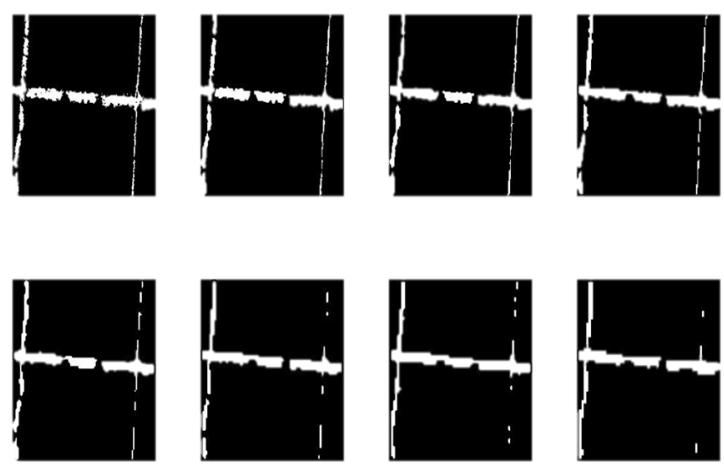

Figure 5. result of multi-resolution morphological algorithm on binary image

\subsection{Long gap coverage:}

3.2.1 Gap parameters: As we said before, the parameters such as occlusion and algorithm weakness caused to remain gaps in a road network.

In this study, distance, momentum, correlation, feature classes and variance along the gap path are introduced as effective parameters in gap detection.

The longer distance between two segments of a road showed lower possibility of gap existence between them.

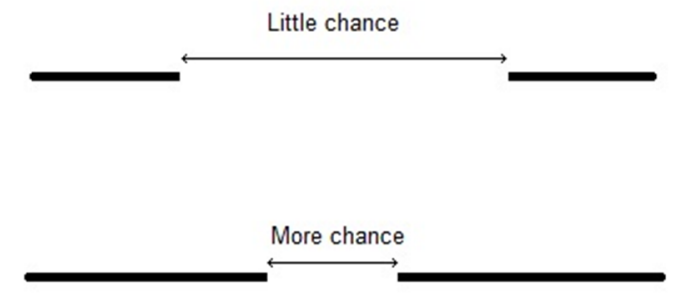

Figure 6. Distance parameter

Whatever the length of the road could be longer, the algorithm that predicts the continuity of the road, works better.

The amount of correlation between continued paths from two segments of a road is a good parameter for assessment the probability of gap existence. In some articles the angle between two continued paths is introduced as one of the correlation parameters (Wittman, et al, 2008). Commonly, the area that results from intersection of tow paths is a good parameter for evaluating the correlation of them. Before, we introduced the distance between two points as one of the correlation parameters. So, in this study, the height of triangle which is made between two end points and the intersection point results from two continued path, is used as correlation parameter.

Basically, the features such as trees, shadows and buildings cover the view of the sensor in respect to road. In this study, the amount of likeness of the features along a road to one of the above feature classes is used for gap detection. If the features along the road belong to one of these classes, we make sure that a gap occurred there.

We used ecognition software for classifying the urban features.

The variance of these feature classes could prevent the gap detection algorithm from making mistakes. The two last parameters (feature and variance along the gap) in compare with other parameters import uncertainty in gap detection process. So the weights of these parameters must be determined, using the resolution and the type of the landscape. These uncertainties result from the weakness of classification algorithm to correctly discriminate the classes along the gap pass.

3.2.2 The fuzzy inference system (FIS): Because the uncertainties in human gap detection methods, we try to develop a system that has an ability to make a correct decision in this situation. As you know, a fuzzy inference system has a sufficient ability to make a decision when we have uncertainties. In this study with importing the gap parameters in a fuzzy inference system, we try to detect the gaps occurrence.

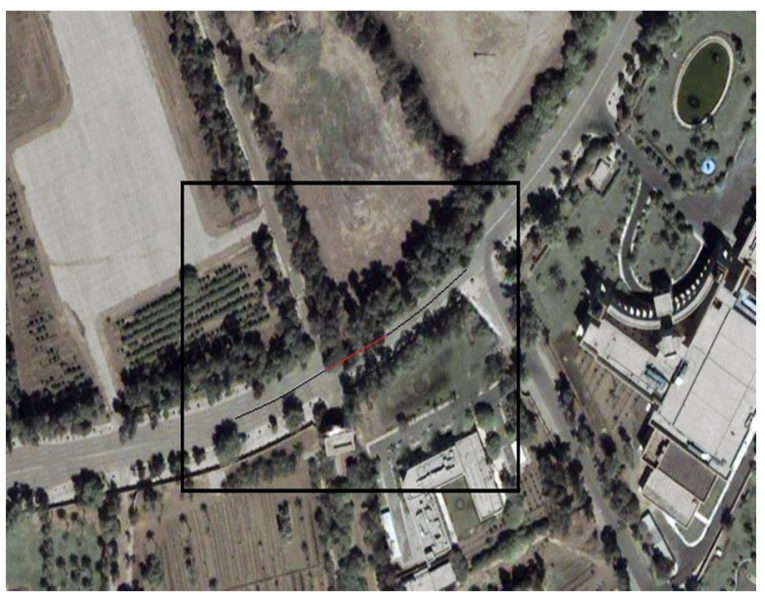

Figure 7. The simulated result for FIS from baghdad

In figure 6 we have tree class across the road network. The FIS can detect that the link hypothesis is true.

\section{ACCURACY ASSESSMENT}

There is no direct approach to evaluate the accuracy of gap detection algorithms. So a reference map of gaps should be produce and use to compare the ability of variant algorithms for gap covering. The results of the algorithm are depending on the type of the landscape and the resolution. Fourth and fifth parameters (features along the gap and variance along the gap) in compare with the others, especially in low and medium resolution, import high uncertainties in algorithm results.

\section{References from Journals:}

Mena, j.b.,2003. State of the art on automatic road extraction for GIS update: a novel classification. Elsevier, pattern recognition letters.

Mashemi, m.,2011a. Automatic covering of little gaps from detected road networks by a multi-resolution morphological approach.

\section{References from Other Literature:}

Wittman,t.,2008.http://www.math.ucla.edu/.Retrieved6,20, 2010,from

http://www.math.ucla.edu/ wittman/reu2008/Reports/ 